\title{
THE STOCHASTIC MECHANICS OF THE PAULI EQUATION
}

\author{
TIMOTHY C. WALLSTROM
}

\begin{abstract}
In stochastic mechanics, the Bopp-Haag-Dankel diffusions on $\mathbb{R}^{3} \times$ $\mathrm{SO}(3)$ are used to represent particles with spin. Bopp and Haag showed that in the limit as the particle's moment of inertia $I$ goes to zero, the solutions of the Bopp-Haag equations converge to that of the regular Pauli equation. Nelson has conjectured that in the same limit, the projections of the Bopp-Haag-Dankel diffusions onto $\mathbb{R}^{3}$ converge to a Markovian limit process. In this paper, we prove this conjecture for spin $\frac{1}{2}$ and regular potentials, and identify the limit process as the diffusion naturally associated with the solution to the regular Pauli equation.
\end{abstract}

\section{INTRODUCTION}

In this paper we consider the stochastic mechanical diffusions associated with three quantum-mechanical equations describing nonrelativistic particles with spin: the Bopp-Haag equations, the regular Pauli equation, and the Pauli equation. Bopp and Haag [1] showed that the solutions to the Bopp-Haag equations converge to the solution of the regular Pauli equation. We will prove the probabilistic counterpart, that the spatial projections of the Bopp-Haag-Dankel diffusions converge to a diffusion naturally associated with the regular Pauli equation.

The Pauli equation is the accepted quantum equation for particles with spin, and was introduced by Wolfgang Pauli in the early days of quantum mechanics. The Bopp-Haag equation was introduced in 1950 by Bopp and Haag [1], who derived the Hamiltonian for a rigidly charged sphere in an electromagnetic field. Assuming the particle has a fixed gyromagnetic ratio equal to that of the electron, the equation has one free parameter, the moment of inertia $I$. We speak of the Bopp-Haag equations in plural to distinguish different choices of $I$.

Bopp and Haag showed that as $I$ went to zero, the solutions $\psi_{I}(t)$ of the Bopp-Haag equations converged to the solution $\psi_{0}(t)$ of an equation with the Pauli Hamiltonian. This equation was not, however, the Pauli equation, since it still employed the regular representation of $\mathrm{SU}(2)$, rather than the irreducible spinor representation. The distinction is important; we shall call the equation

Received by the editors August 10, 1988.

1980 Mathematics Subject Classification (1985 Revision). Primary 81C20, 60J60; Secondary $60 \mathrm{G} 17$. tion.

Key words and phrases. Stochastic mechanics, spin diffusions, Bopp-Haag, Dankel, Pauli equa- 
with the Pauli Hamiltonian and the regular representation space the "regular Pauli equation."

In 1970, Dankel [3] introduced the stochastic mechanical diffusions associated with the Bopp-Haag equations. These were diffusions on $\mathbb{R}^{3} \times \mathrm{SO}(3)$, and provided a satisfying picture of a diffusing particle with the classical attributes of definite position and orientation. As we will see, we can also associate diffusions with solutions to the regular Pauli and (spinor) Pauli equations, although these diffusions describe the particle's motion on $\mathbb{R}^{3}$ alone.

The purpose of this paper is to prove that the spatial projections of the diffusions associated to the Bopp-Haag equations converge, as $I \rightarrow 0$, to a limiting diffusion. The existence of such a limit was conjectured by Nelson in $[7, \mathrm{p}$. 107]. We prove this conjecture for smooth spin- $\frac{1}{2}$ wavefunctions and regular potentials. We will also identify the limit process as the diffusion naturally associated with the regular Pauli equation. When the density evolutions of the regular and spinor Pauli equations coincide, this limit process is the same as the diffusion process naturally associated with the Pauli equation.

There are two main parts to the proof. The first is to show that as $I \rightarrow 0$, the spatial drift, which depends on both the spatial and orientational coordinates, can be approximated by its orientational average. This is because the orientational motion is ergodic, and the relaxation time is proportional to $I$. The second part of the proof is to show that as $I \rightarrow 0$ these orientationally averaged drifts converge, and that this in turn implies the convergence of the diffusions.

In $\S 2$, we present the Hamiltonians we will be studying, and discuss their interrelations. In $\S 3$, we indicate how to construct the associated diffusions. In $\S 4$, we prove that the orientationally averaged drift coefficients of a diffusion on $\mathbb{R}^{3} \times \mathrm{SO}(3)$ describe a process with the spatially projected density, a result which will be essential to the proof of the conjecture. The two central parts of the proof just discussed are treated in $\S 5$ and $\S 6$, respectively. In particular, the proof of Nelson's conjecture for spin- $\frac{1}{2}$ is completed in $\S 6$. In $\S 7$, we identify the limit diffusion and explore its connection to the regular and spinor Pauli equations.

\section{SPIN EQUATIONS}

We present the Bopp-Haag, regular Pauli, and (spinor) Pauli equations, and discuss briefly their analytic relationships.

Let $M=\mathbb{R}^{3} \times \mathrm{SO}(3)$, and denote its covering space by $\widetilde{M}=\mathbb{R}^{3} \times \mathrm{SU}(2)$. Reserve the letters $x, y$, and $z=(x, y)$ for elements of $\mathbb{R}^{3}, \mathrm{SO}(3)$ or $\mathrm{SU}(2)$, and $M$ or $\tilde{M}$, respectively; reserve their Greek counterparts $\xi, \eta$, and $\zeta$, for diffusion processes on $\mathbb{R}^{3}, \mathrm{SO}(3)$ and $M$. Let $L_{i}$, for $i=1,2,3$, be a basis for the Lie algebra on $\mathrm{SU}(2)$, and its projection to $\mathrm{SO}(3)$. Recall that $L^{2}(\mathrm{SU}(2))=\bigoplus_{n=0}^{\infty} \mathscr{H}^{n / 2}$, where $\mathscr{H}^{s}$, the spin- $s$ subspace, is an eigenspace of the Laplacian with eigenvalue $s(s+1)$. Define $\mathscr{K}^{s}$ as $L^{2}\left(\mathbb{R}^{3}\right) \otimes \mathscr{H}^{s}$. Then $L^{2}(\widetilde{M})=\bigoplus_{n=0}^{\infty} \mathscr{K}^{n / 2}$. 
Let $H_{I}$, the Bopp-Haag Hamiltonian for a particle with moment of inertia $I>0$, be given by

$$
H_{I}=\frac{1}{2}\left(\frac{1}{i} \nabla^{i}-A^{i}\right)\left(\frac{1}{i} \nabla_{i}-A_{i}\right)+\frac{1}{2 I}\left(\frac{1}{i} L^{i}-I B^{i}\right)\left(\frac{1}{i} L_{i}-I B_{i}\right)+\Phi
$$

(repeated indices summed), and let $H_{0}$, the Pauli Hamiltonian, be given by

$$
H_{0}=\frac{1}{2}\left(\frac{1}{i} \nabla^{i}-A^{i}\right)\left(\frac{1}{i} \nabla_{i}-A_{i}\right)+i B_{i} L^{i}+\Phi,
$$

where we denote the scalar potential by $\Phi(x)$, the vector potential by $A(x)$, and the magnetic field by $B(x)$. (For simplicity we assume that $\Phi, A$, and $B$ are time-independent.) Let $\psi_{I}(t)$ be the solution of

$$
i \frac{\partial \psi_{I}}{\partial t}(t)=H_{I} \psi_{I}(t)
$$

with initial condition $\psi_{I}(x, y, 0)=\psi^{0}(x, y)$. For $I>0$, this is the BoppHaag equation; for $I=0$, this is the regular Pauli equation, also known as the Dankel equation [1, 3, 7]. If we consider the Hamiltonian $H_{0}$ acting on the state space of spin- $s$ spinors, and if we replace $i L^{i}$ by the appropriate rotational generator for this representation, we obtain the Pauli equation. We will be mostly concerned with the spin- $-\frac{1}{2}$ diffusions; in this case $i L^{i}$ becomes $\frac{1}{2} \sigma^{i}$, where the $\sigma^{i}$ are the Pauli spin matrices.

Let

$$
H_{I}^{\prime}=H_{I}+\frac{1}{2 I} L^{i} L_{i},
$$

and denote the solutions to the corresponding equation by $\psi_{I}^{\prime}(t)$. If $\psi^{0} \in \mathscr{K}^{s}$, for some $s$, then $\psi_{I}$ and $\psi_{I}^{\prime}$ will lead to the same drift coefficients, and under mild regularity assumptions on the potentials, $\psi_{I}^{\prime} \rightarrow \psi_{0}$ in $L^{2}$ as $I \rightarrow 0$ [1, 3]. In other words, once a large constant phase is removed, the solutions to the Bopp-Haag equations converge to the solution of the regular Pauli equation.

The regular spin-s Pauli equation, in turn, is a $(2 s+1)$-fold copy of the usual spinor Pauli equation. This follows from well-known facts concerning the regular and spinor representations; see, e.g., [4] or [8]. Assuming that the irreducible subspaces are represented as columns, the density evolution of a regular Pauli wavefunction can be duplicated by that of a Pauli spinor if and only if each column in the regular wavefunction is parallel to the same Pauli spinor.

The Bopp-Haag and regular Pauli wavefunctions have been defined on $\mathbb{R}^{3} \times$ $\mathrm{SU}(2)$, so how can we define associated diffusions on $\mathbb{R}^{3} \times \mathrm{SO}(3)$ ? The wavefunctions we consider will always be in fixed spin states, for which $\psi(x, y, t)=$ $\pm \psi(x,-y, t)$. In such cases, the density and drift coefficients will be singlevalued on $\mathbb{R}^{3} \times \mathrm{SO}(3)$. 


\section{SPIN DIFFUSIONS}

We now consider the definition of the stochastic-mechanical diffusions associated with these spin equations. The usual foundational formulations of stochastic mechanics lead to Hamiltonians whose form is that of a generalized Schrödinger operator with electromagnetic interaction. They will not work, therefore, for the regular or spinor Pauli equations, nor is it clear from this perspective that diffusion processes can be associated in a natural way to the solutions of such equations. For this reason, we briefly review a fairly unambiguous procedure for associating a diffusion process to nearly any nonrelativistic quantum equation. This procedure, which is heuristic rather than systematic or foundational, yields the same diffusions as are usually selected out by the more foundational formulations when applicable. (For the latgter, cf. [7, 11].)

Consider the continuity equation expressing conservation of probability density for the quantum equation in question:

$$
\frac{\partial \rho}{\partial t}=-\nabla \cdot(v \rho)
$$

This can be rewritten as a Fokker-Planck equation:

$$
\frac{\partial \rho}{\partial t}=-\nabla \cdot[(v+u) \rho]+\frac{\nu}{2} \Delta \rho,
$$

where $u$, the osmotic velocity, is defined as $(\nu / 2) \nabla \log \rho$. We can therefore associate with a solution to any such quantum equation a diffusion process $\xi(t)$ with drift $b=v+u$ and covariance $\nu$, provided the diffusion exists. The process $\xi(t)$ is a solution to the Itô stochastic differential equation

$$
d \xi(t)=b(\xi(t), t) d t+d w(t),
$$

where $w(t)$ is the Wiener process with covariance $\nu$. Carlen [2] has proved the existence of such diffusions under the very weak finite energy condition:

$$
\int_{0}^{T} \int\left(u^{2}+v^{2}\right) \rho d x d t<\infty .
$$

The backward process (with increments $d_{*} \xi(t)=\xi(t)-\xi(t-d t)$ ) has drift $b_{*}=v-u$; see [7] for a proof and more details.

Although any value of $\nu$ will lead to the correct density evolution, we will choose $\nu$ so that the coefficient of the Laplacian in the Fokker-Planck equation is equal to the coefficient of the Laplacian in the associated quantum equation (cf. [5]).

As an example, consider the Schrödinger equation with electromagnetic interaction. This procedure yields

$$
v^{i}=\operatorname{Im} \frac{\nabla^{i} \psi}{\psi}-A^{i}, \quad u^{i}=\operatorname{Re} \frac{\nabla^{i} \psi}{\psi}, \quad \nu=1 .
$$

(To simplify our equations, we have tacitly chosen units such that $\hbar, m$, and $c$ are equal to 1 , and absorbed the charge $e$ into the vector potential.) 
The diffusions associated with the Bopp-Haag equations were defined by Dankel in [3]. His derivation was based on a generalization of Nelson's original formulation of stochastic mechanics to the context of a Riemannian manifold, but it leads to the same diffusions as the heuristic procedure just described. Note that the coefficient of the angular Laplacian is $I^{-1}$, so the orientational part of the generator scales as $I^{-1}$. Also, as shown in [10], the spin- $\frac{1}{2}$ diffusions are uniformly ergodic. These are the only facts we need to know about the orientational process, and these results are used only once, in Theorem 3.

We will denote the process associated with the solution $\psi_{I}(x, y, t)$ of the Bopp-Haag equation with moment of inertia $I$ by $\zeta_{I}(t)=\left(\xi_{I}(t), \eta_{I}(t)\right)$. We will always consider the processes $\zeta_{I}(t)$ to be constructed from the Wiener process by means of the Itô stochastic integral [6]. We define the $\zeta_{I}(t)$ on the probability space $\left(\Omega, \mathscr{P}_{T}, \operatorname{Pr}\right)$, where $\Omega=M \times W$, with typical element $\omega=(z, w)$. Here, $W$ is the space of continuous paths $w(t)=\left(w_{1}(t), w_{2}(t)\right)$ from $[0, T]$ to $M, \mathscr{P}_{t}=\sigma\{z(t): s \in[0, t]\}$, where $z(t)=z+w(t)$, and $\operatorname{Pr}=\rho^{0}(z) d z \otimes \mathbf{W}$, where $\mathbf{W}$ is Wiener measure and $\rho^{0}(z)=\left|\psi^{0}(z)\right|^{2}$. (We keep the initial condition fixed as we vary $I$.) Thus, if $\omega=(z, w)$,

$$
\zeta_{I}(t)(\omega)=\zeta_{I}(0)+\int_{0}^{t} b_{I}\left(\zeta_{I}(s), s\right) d s+w(t),
$$

where $\zeta_{I}(0)=z$, and $b_{I}$ is the drift associated to $\psi_{I}$. (This is only formal since $M$ is a Riemannian manifold; see [6] or [7] for a more detailed treatment.) We denote the expectation with respect to $\operatorname{Pr}$ by $\mathbf{E}$. To distinguish the spatial and orientational components of the drift, we will use a numerical subscript, as in $b_{I, 1}$ or $b_{I, 2}$.

The diffusions corresponding to the (spinor) Pauli equation fit neatly into the formalism described above. If $\psi_{i}$ is a Pauli spinor, the current and osmotic velocities are easily calculated to be

$$
v=\frac{1}{\rho} \sum_{i} \operatorname{Im}\left(\frac{\nabla \psi_{i}}{\psi_{i}}\right) \rho_{i}=\frac{1}{\rho} \sum_{i} v_{i} \rho_{i}, \quad u=\frac{\nabla \rho}{2 \rho}=\frac{1}{\rho} \sum_{i} u_{i} \rho_{i} .
$$

This is a diffusion process on $\mathbb{R}^{3}$. We will assume that this and related $\mathbb{R}^{3}$ diffusions are defined on the same space and in the same way as the Bopp-HaagDankel diffusions, and constructed from Wiener measure, though naturally the path $\xi(t)$ will not depend on the orientational component of the Wiener path.

The regular Pauli equation does not lend itself to the procedure described in this section, because the wavefunction has an orientational dependence but no orientational Laplacian, and we thus have no way of determining the orientational covariance or drifts. We can however define a spatial drift $b_{0,1}$ just as we define the spatial drifts $b_{I, 1}$ of the Bopp-Haag equations, and this quantity will arise naturally in the sequel. Diffusion processes associated to the regular Pauli equation will be discussed in the final section of this paper. 


\section{A RESUlt ON THE PROJECTED DENSITY}

Suppose we have a diffusion process on $M=M_{1} \times M_{2}$ with density $\rho(x, y, t)$, and we project this density onto $M_{1}$. In other words, consider the density

$$
\hat{\rho}(x, t)=\int_{M_{2}} \rho(x, y, t) d y .
$$

Does $\hat{\rho}(x, t)$ describe the density evolution of a diffusion, and if so, do its coefficients bear any simple or useful relation to the diffusion coefficients of the original process? The answer is yes, as the theorem of this section will show. This result will be essential to proving Nelson's conjecture.

For any (real) function $f(x, y, t)$ on $M \times[0, T]$, let

$$
\begin{aligned}
& \bar{f}(x, t)=\frac{1}{\hat{\rho}(x, t)} \int_{M_{2}} \rho(x, y, t) f(x, y, t) d y, \\
& f^{\perp}(x, y, t)=f(x, y, t)-\bar{f}(x, t)
\end{aligned}
$$

whenever $\hat{\rho}(x, t)>0$, and zero elsewhere.

Theorem 1. Suppose $\rho$ and $v$ are the density and current velocity of a diffusion on $M=M_{1} \times M_{2}$, where $\partial M_{2}=\varnothing$. Then

$$
\frac{\partial \hat{\rho}}{\partial t}=-\nabla_{1} \cdot\left(\bar{v}_{1} \hat{\rho}\right),
$$

and $\hat{u}=\bar{u}_{1}$ (where $\hat{u}$ is defined as $\frac{1}{2} \nabla \log \hat{\rho}$ ).

Proof. The continuity equation for $\rho$ and $v$ is

$$
\frac{\partial \rho}{\partial t}=-\nabla_{1} \cdot\left(v_{1} \rho\right)-\nabla_{2} \cdot\left(v_{2} \rho\right)
$$

The equation in $\hat{\rho}$ is obtained by integrating this equation over $M_{2}$. The relation $\hat{u}=\bar{u}_{1}$ is obtained by substituting $\nabla_{1} \rho / 2 \rho$ into the defining relation for $\bar{u}_{1}$.

We note also that

$$
\bar{u}_{1}^{2}+\bar{v}_{1}^{2} \leq \overline{u_{1}^{2}+v_{1}^{2}},
$$

so the "energy" of the new drift coefficients is less than that of the original coefficients. The condition $\partial M_{2}=\varnothing$ is taken just to make certain the second divergence term evaporates; we can dispense with it by proving the result for the weak continuity equation instead [2].

Recalling the procedure of the previous section, we see that a diffusion with forward drift $\bar{u}_{1}(x, t)+\bar{v}_{1}(x, t)$ and initial density $\hat{\rho}(x, 0)$ will evolve with density $\hat{\rho}(x, t)$. We call this the process with the averaged drifts.

The bar notation will be used frequently to indicate the orientational average of the spatial drifts $b_{I, 1}$ and $b_{0,1}$. When we write $\bar{b}_{I, 1}$ for $I \geq 0$, it will always be assumed that $b_{I, 1}$ is being averaged over the density of the wavefunction from which it was derived. 


\section{THE ERGODIC LIMIT OF THE SPATIAL DRIFT}

Our objective in this section is to prove the following:

Theorem 2. Assume that $\psi^{0} \in \mathscr{K}^{1 / 2}$ has finite energy, that $\psi_{I}(t)$ is the solution to the Bopp-Haag equation with moment of inertia $I>0$ and initial condition $\psi^{0}$, and that for some small $I_{0},(t, I) \mapsto\left\|\psi_{I}(t)\right\|$ is jointly continuous from $[0, T] \times\left[0, I_{0}\right]$ into $\mathbb{R}$. Let $\zeta_{I}(t)=\left(\xi_{I}(t), \eta_{I}(t)\right)$ be the Bopp-Haag-Dankel diffusion corresponding to the wavefunction $\psi_{I}(x, y, t)$. Then

$$
\sup _{t \in[0, T]}\left|\xi_{I}(t)-\xi_{I}(0)-\int_{0}^{t} \bar{b}_{I, 1}\left(\xi_{I}(s), s\right) d s-w_{1}(t)\right| \rightarrow 0
$$

in probability as $I \rightarrow 0$.

This theorem shows that as $I \rightarrow 0$, the effect of the orientational motion is averaged out, and the spatial drift term depends only on the spatial part of the diffusion.

We begin by proving two theorems which show, roughly speaking, that the solutions to the diffusion equation become constant in the orientational component arbitrarily rapidly, as $I \rightarrow 0$. The constant $\lambda$ is defined as the infimum of the relaxation constants of the orientational generators of the spin- $-\frac{1}{2}$ diffusions. The relaxation constant of the generator, in turn, is defined as the infimum of the eigenvalues of the set of eigenfunctions perpendicular to the constant function 1. It gives a quantitative measure of the rate at which the orientational diffusions relax to their ergodic limits. The number $\lambda$ is greater than zero; for a proof, see [10]. We write the solutions to the backward (forward) diffusion equation, with initial (final) condition $f(x)$ at time $t$, as $P_{*}(s, t) f(x)$ $(P(s, t) f(x))$ or more briefly, as $P_{*} f(x, s) \quad(P f(x, s))$, where the $t$-argument is assumed. These are equal to $\mathbf{E}^{x, s}[f(\xi(t))]$ for $s>t$ and $s<t$, respectively, where $\mathbf{E}^{x, s}[\cdot]$ is the conditional expectation $\mathbf{E}[\cdot \mid \xi(s)=x]$.

Theorem 3. Let $f$ be an element of $L^{2}\left(\rho_{s}\right)$. Then

$$
\int_{s}^{t}\left\|\left(P_{*}^{I} f\right)^{\perp}\right\|_{r} d r \leq(t-s)^{1 / 2}\left(\frac{I}{2 \lambda}\right)^{1 / 2}\|f\|_{s} .
$$

Proof. Write $F(z, r)=P_{*}^{I} f(z, r)=P_{*}^{I}(r, s) f(z)$. Then

$$
\begin{aligned}
\frac{d}{d r}\|F\|_{r}^{2} & =-\left\|\nabla_{1} F\right\|_{r}^{2}-\frac{1}{I}\left\|\nabla_{2} F\right\|_{r}^{2} \\
& \leq \frac{1}{I}\left(F, \Delta_{\rho, 2} F\right)_{r} \leq-\frac{2 \lambda}{I}\left\|F^{\perp}\right\|_{r}^{2} .
\end{aligned}
$$

$\left(\Delta_{\rho, 2} \equiv \Delta+2 u_{2} \cdot \nabla\right.$. For the first and third relations, cf. [10].) But

so

$$
\int_{s}^{t} \frac{d}{d r}\|F\|_{r}^{2} d r \geq-\|F\|_{s}^{2},
$$

$$
\int_{s}^{t}\left\|F^{\perp}\right\|_{r}^{2} d r \leq \frac{I}{2 \lambda}\|F\|_{s}^{2} .
$$


By Hölder's inequality,

$$
\int_{s}^{t}\left\|F^{\perp}\right\|_{r} d r \leq(t-s)^{1 / 2}\left(\frac{I}{2 \lambda}\right)^{1 / 2}\|F\|_{s} .
$$

Theorem 4. Let $f(z, s)$ be given, and suppose $\|f\|_{s}<K$, for some $K>0$, for $s \in[0, T]$. Then

$$
\left\|\int_{0}^{t} f^{\perp}\left(\zeta_{I}(s), s\right) d s\right\| \leq 2 K\left(\frac{2 I t^{3}}{9 \lambda}\right)^{1 / 4} .
$$

Proof. It will be convenient to indicate the time argument of $f$ by a subscript. We will also drop the $I$ subscripts, since a fixed $I$ will be assumed.

Note that $\bar{f}^{2} \leq \overline{f^{2}}$, so $\|\bar{f}\|_{s} \leq\|f\|_{s}$, and $\left\|f^{\perp}\right\|_{s} \leq 2\|f\|_{s} \leq 2 K$. Now we calculate:

$$
\begin{aligned}
& \mathbf{E}\left[\int_{0}^{t} f_{s}^{\perp}(\zeta(s)) d s \int_{0}^{t} f_{u}^{\perp}(\zeta(u)) d u\right] \\
& \quad=\int_{0}^{t} d u \mathbf{E}\left[\int_{0}^{u} f_{s}^{\perp}(\zeta(s)) d s+\int_{u}^{t} f_{s}^{\perp}(\zeta(s)) d s\right] f_{u}^{\perp}(\zeta(u)) \\
& \quad=\int_{0}^{t} d u \int_{0}^{u} d s\left(P_{*}\left(f_{s}^{\perp}\right), f_{u}^{\perp}\right)_{u}+\int_{0}^{t} d u \int_{u}^{t} d s\left(P\left(f_{s}^{\perp}\right), f_{u}^{\perp}\right)_{u} \\
& \quad=\int_{0}^{t} d s \int_{s}^{t} d u\left(P_{*}\left(f_{s}^{\perp}\right), f_{u}^{\perp}\right)_{u}+\int_{0}^{t} d s \int_{0}^{s} d u\left(P\left(f_{s}^{\perp}\right), f_{u}^{\perp}\right)_{u} .
\end{aligned}
$$

Now

$$
\left.\overline{\left(P_{*}\left(f_{s}^{\perp}\right)\right.}, f_{u}^{\perp}\right)_{u}=\left(\overline{P_{*}\left(f_{s}^{\perp}\right)}, \overline{f_{u}^{\perp}}\right)_{u}=0
$$

so

$$
\left(P_{*}\left(f_{s}^{\perp}\right), f_{u}^{\perp}\right)_{u}=\left(\left(P_{*}\left(f_{s}^{\perp}\right)\right)^{\perp}, f_{u}^{\perp}\right)_{u} .
$$

Furthermore,

$$
\begin{gathered}
\int_{0}^{t} d s \int_{s}^{t} d u\left(\left(P_{*}\left(f_{s}^{\perp}\right)\right)^{\perp}, f_{u}^{\perp}\right)_{u} \leq \int_{0}^{t} d s \int_{s}^{t} d u\left\|\left(P_{*}\left(f_{s}^{\perp}\right)\right)^{\perp}\right\|_{u}\left\|f_{u}^{\perp}\right\|_{u} \\
\leq(2 K)^{2}\left(\frac{I}{2 \lambda}\right)^{1 / 2} \int_{0}^{t}(t-s)^{1 / 2} d s=(2 K)^{2}\left(\frac{I}{2 \lambda}\right)^{1 / 2} \frac{2}{3} t^{3 / 2} .
\end{gathered}
$$

The second term can be bounded in the same manner.

Proof of Theorem 2. For any function $f(z, s), M(t) \equiv \int_{0}^{t} f^{\perp}\left(\zeta_{I}(s), s\right) d s$ is a $\mathscr{P}_{t}$-martingale. Thus $M(t)^{2}$ is a $\mathscr{P}_{t}$-submartingale. Let $f(z, s)=b_{I, 1}(z, s)$. Then, by Doob's submartingale inequality,

$$
\operatorname{Pr}\left\{\sup _{0 \leq t \leq T}\left|\int_{0}^{t} b_{I, 1}^{\perp}\left(\zeta_{I}(s), s\right) d s\right| \geq \varepsilon\right\} \leq \frac{\left\|\int_{0}^{T} b_{I, 1}^{\perp}\left(\zeta_{I}(s), s\right) d s\right\|^{2}}{\varepsilon^{2}} .
$$

By continuity, there exists $K>0$ such that for $(t, I) \in[0, T] \times\left[0, I_{0}\right]$,

$$
\left\|b_{I, 1}\right\|_{t}<2\left\|\nabla \psi_{I}(t)\right\|<K \text {. }
$$


The theorem follows from the bound in Theorem 4, and the fact that

$$
\xi_{I}(t)=\xi_{I}(0)+\int_{0}^{t} b_{I, 1}\left(\zeta_{I}(s), s\right) d s+w_{1}(t)
$$

\section{NELSON'S CONJECTURE}

To complete the proof of Nelson's conjecture, we must show that

$$
\sup _{t \in[0, T]}\left|\int_{0}^{t} \bar{b}_{I, 1}\left(\xi_{I}(s), s\right) d s-\int_{0}^{t} \bar{b}_{0,1}\left(\xi_{I}(s), s\right) d s\right| \rightarrow 0
$$

in some sense, which together with Theorem 2 implies that

$$
\sup _{t \in[0, T]}\left|\xi_{I}(t)-\xi_{I}(0)-\int_{0}^{t} \bar{b}_{0,1}\left(\xi_{I}(s), s\right) d s-w_{1}(t)\right| \rightarrow 0
$$

in some sense. We must then deduce from this that $\xi_{I} \rightarrow \xi$, where $\xi(t)$ is the diffusion on $\mathbb{R}^{3}$ with initial density $\hat{\rho}^{0}(x)=\int_{\mathrm{SO}(3)}\left|\psi^{0}(x, y)\right|^{2} d y$ and drift $\bar{b}_{0,1}(x, t)$.

It is easy to infer convergence of $\xi_{I}$ to $\xi$ from (29) if $\bar{b}_{0,1}$ satisfies a uniform Lipschitz condition. The most characteristic feature of stochastic mechanical diffusions, however, is that the drifts are generally singular, and will not satisfy such a condition. Furthermore, (28) will not hold when $\xi_{I}(t)$ enters regions where $\bar{b}_{0,1}$ diverges. The finite-energy bounds on nodal approach in [9] provide a way around these problems. We choose a compact subset $D$ of $\mathbb{R}^{3} \times[0, T]$ in the complement of the nodes of $\hat{\rho}_{0}(x, t)$, and discard the paths not contained in $D$, being assured by our finite-energy estimates that by choosing $D$ properly the measure of the discarded paths can be made arbitrarily small. We then use the fact that $\bar{b}_{0,1}$ is bounded and uniformly Lipschitz on $D$ to infer that on this set (28) holds and $\xi_{I} \rightarrow \xi$.

There are two problems with this approach. First, the nodal sets will shift as $\xi_{I} \rightarrow \xi_{0}$. How do we know that $\xi_{I}$ will stay away from the nodes of $\hat{\rho}_{0}$ ? Second, since we plan to make such heavy use of the finite-energy estimates, it is worth noting in advance that as $I \rightarrow 0$, the energy integral diverges. Indeed, $\rho_{I}(x, \cdot, t)$ has nodes for every $x$, and $\zeta_{I}(t)$ will, in its ergodic zeal, visit the set $\left\{\rho_{I}<\varepsilon\right\}$ for any $\varepsilon>0$ with arbitrarily high frequency as $I \rightarrow 0$. The culprit is the orientational part of the energy, which is proportional to $1 / I$.

To deal with the first problem, we anchor the nodal sets by adopting strong regularity assumptions ensuring that $\hat{\rho}_{I} \rightarrow \hat{\rho}_{0}$ in the uniform norm. By avoiding its own nodal sets, $\xi_{I}$ avoids those of $\xi_{0}$. The second problem is resolved by Theorem 1, which indicates that the projected density and the averaged drifts obey a continuity equation. This makes it possible to bound the approach to the nodes of the projected density in terms of the energy of the averaged spatial drifts, which does stay bounded. 
Theorem 5 (Nelson's conjecture). Assume that $\psi^{0}, \boldsymbol{\Phi}, A$, and $B$ are smooth functions of compact support, that $\psi^{0}$ is a spin- $\frac{1}{2}$ wavefunction, and that $\psi_{I}(x, y, t)$ is the solution to the corresponding Bopp-Haag equation with moment of inertia $I>0$ and initial condition $\psi_{I}(x, y, 0)=\psi^{0}(x, y)$. Let $\zeta_{I}(t)$ be the corresponding Bopp-Haag-Dankel diffusion, and $\xi_{I}(t)$ its spatial component. Let $\xi(t)$ be the diffusion on $\mathbb{R}^{3}$ with initial density $\hat{\rho}^{0}(x)$ and drift $\bar{b}_{0,1}(x, t)$. Then

$$
\sup _{t \in[0, T]}\left|\xi_{I}(t)-\xi(t)\right| \rightarrow 0
$$

in probability as $I \rightarrow 0$.

Proof. Before embarking on the main part of the proof, we need to establish that $\psi_{I}^{\prime} \rightarrow \psi_{0}$ in a strong sense. We will show that under the conditions stated in the theorem, $\hat{\rho}_{I}$ converges uniformly to $\hat{\rho}_{0}$, and that the orientationally averaged spatial drifts $\bar{u}_{I, 1}$ and $\bar{v}_{I, 1}$ converge uniformly to $\bar{u}_{0,1}$ and $\bar{v}_{0,1}$ away from the nodes of $\hat{\rho}_{0}$.

Let $W_{m}$, the $m$ th Sobolev space, be defined by

$$
W_{m}=\left\{f:(1-\Delta)^{m / 2} f \in L^{2}(\widetilde{M})\right\} .
$$

Under the stated assumptions, we can construct unitary groups $\exp \left(-i t H_{I}^{\prime}\right)$ and $\exp \left(-i t H_{0}\right)$ on any $W_{m}$, and $H_{I}^{\prime}-H_{0}$ is an $H_{0}$-bounded perturbation on $H_{0}$, analytic in $I$, for any $W_{m}$. Therefore, given $\psi^{0}, \psi_{I}^{\prime}(t)$ is a jointly continuous mapping of $I$ and $t$ into $W_{m}$, for all $m$. In particular, as $I \rightarrow 0, \psi_{I}^{\prime}(t)$ converges to $\psi_{0}(t)$ in $W_{m}$, for all $m$. Since the uniform norm is bounded by a constant times a Sobolev norm of some finite order depending only on the dimension, and since $\|\nabla \psi\|_{m} \leq\|\psi\|_{m+1}$, we obtain uniform convergence of $\psi_{I}^{\prime}$ and $\nabla \psi_{I}^{\prime}$ to $\psi_{0}$ and $\nabla \psi_{0}$, respectively.

Since $\psi^{0}$ is in a definite spin state, $\psi_{I}^{\prime}$ and $\psi_{I}$ differ only by a phase, and the drifts calculated from $\psi_{I}^{\prime}$ will equal those calculated from $\psi_{I}$. Since

$$
\left(\nabla_{1} \psi_{I}^{\prime}\right) \psi_{I}^{\prime *}-\left(\nabla_{1} \psi_{0}\right) \psi_{0}^{*}=\left(\nabla_{1} \psi_{I}^{\prime}-\nabla_{1} \psi_{0}\right) \psi_{I}^{\prime *}+\left(\nabla_{1} \psi_{0}\right)\left(\psi_{I}^{\prime *}-\psi_{0}^{*}\right),
$$

$\left(u_{I, 1}+i v_{I, 1}\right) \rho_{I}$ converges uniformly to $\left(u_{0,1}+i v_{0,1}\right) \rho_{0}$ (where we are bounding the norm of $\nabla_{1} \psi_{I}^{\prime}-\nabla_{1} \psi_{0}$ by that of $\left.\nabla \psi_{I}^{\prime}-\nabla \psi_{0}\right)$. Integrating over $\operatorname{SO}(3)$, we find that as $I \rightarrow 0$,

$$
\left(\bar{u}_{I, 1}+i \bar{v}_{I, 1}\right) \hat{\rho}_{I} \rightarrow\left(\bar{u}_{0,1}+i \bar{v}_{0,1}\right) \hat{\rho}_{0} \quad \text { and } \quad \hat{\rho}_{I} \rightarrow \hat{\rho}_{0}
$$

uniformly. In the $I \rightarrow 0$ limit, we thus obtain uniform convergence of $\bar{u}_{I, 1}$ to $\bar{u}_{0,1}$ and $\bar{v}_{I, 1}$ to $\bar{v}_{0,1}$ away from the nodes of $\hat{\rho}_{0}$.

Since $\psi_{I}^{\prime} \rightarrow \psi_{0}$ in $W_{1}$,

$$
A_{I, 1}=\int_{0}^{T} \int\left(u_{I, 1}^{2}+v_{I, 1}^{2}\right) \rho_{I} d z d t
$$

goes to $A_{0,1}$ as $I \rightarrow 0$. In particular, there exist numbers $C$ and $I_{0}$ such that for all $I<I_{0}, A_{I, 1}<C$. We will assume henceforth that $I<I_{0}$. 
The nodes result of [9] can easily be adapted to show that $\xi_{I}(t)$ stays away from the nodes of $\hat{\rho}_{I}(x, t)$. Simply calculate $d \bar{R}_{I}\left(\zeta_{I}(t), t\right)$ instead of $d R_{I}\left(\zeta_{I}(t), t\right)$, keeping in mind that by Theorem $1, \bar{R}_{I}$ and $\bar{S}_{I}$ satisfy the "continuity equation in $R$ and $S$ ":

$$
\partial \bar{R}_{I} / \partial t+\nabla \bar{R}_{I} \cdot \nabla \bar{S}_{I}+\frac{1}{2} \Delta \bar{S}_{I}=0 .
$$

$\left(R_{I}\right.$ and $S_{I}$ are defined formally by $\psi_{I}=\exp \left(R_{I}+i S_{I}\right)$; see [9] for more details.) Also, it is easy to show that for any $\varepsilon>0$, there exists $d>0$ such that $\xi_{I}(t)$ stays within a sphere of radius $d$ with probability greater than $1-\varepsilon$. In both cases, the bounds depend only on $A_{I, 1}$ and $\psi^{0}$, and we thus obtain uniform bounds for $I<I_{0}$.

Choose $\delta$ and $d$ such that for all $I<I_{0}$,

$$
\operatorname{Pr}\left\{\left(\xi_{I}(t), t\right) \in \mathbb{R}^{3} \times[0, T] \backslash \hat{Z}_{2 \delta}^{I}, \forall t \in[0, T]\right\}>1-\varepsilon / 4,
$$

and

$$
\operatorname{Pr}\left\{\left|\xi_{I}(t)\right|<d, \forall t \in[0, T]\right\}>1-\dot{\varepsilon} / 4,
$$

where

$$
\hat{Z}_{\delta}^{I}=\left\{(x, t): \hat{\rho}_{I}(x, t) \leq \delta\right\} .
$$

Choose $I_{0}^{\prime}<I_{0}$ such that for $I<I_{0}^{\prime}$,

$$
\left\|\hat{\rho}_{I}-\hat{\rho}_{0}\right\|_{\infty}<\delta .
$$

Let

$$
V_{d}=\{x:|x| \leq d\}, \quad D=V_{d} \times[0, T] \backslash \hat{Z}_{\delta}^{0},
$$

and

$$
\Lambda_{I}=\left\{\omega:\left(\xi_{I}(t), t\right) \in D, \forall t \in[0, T]\right\} .
$$

Then $\operatorname{Pr}\left\{\Lambda_{I}\right\}>1-\varepsilon / 2$ for any $I<I_{0}^{\prime}$, since $\hat{Z}_{\delta}^{0} \subset \hat{Z}_{2 \delta}^{I}$. Note that $D$ is independent of $I$.

Let

$$
K=\sup _{(x, t) \in D}\left|\nabla \cdot \bar{b}_{0,1}(x, t)\right| \text { and } B=\sup _{(x, t) \in D}\left|\bar{b}_{0,1}(x, t)\right| .
$$

Then $K<\infty$ and $B<\infty$ because $D$ is compact and under our assumptions $\psi$ is smooth and $|\psi|>0$ on $D$.

The drift $\bar{b}_{0,1}$ satisfies a uniform Lipschitz condition with constant $K$ on $D$. Suppose $\varepsilon>0$ is given. Then I claim that if $x(0)=\xi(0),(x(t), t) \in D$ for all $t$ in $[0, T]$ and

$$
\sup _{t \in[0, T]}\left|x(t)-x(0)-\int_{0}^{t} \bar{b}_{0,1}(x(s), s) d s-w_{1}(t)\right|<\varepsilon_{0},
$$

then

$$
\sup _{t \in[0, T]}|x(t)-\xi(t)|<\varepsilon_{0} e^{K T}
$$


Let $\beta<1$, and define $h$ by $\beta=h K$. Now

$$
\xi(t)=\xi(0)+\int_{0}^{t} \bar{b}_{0.1}(\xi(s), s) d s+w_{1}(t),
$$

so

$$
\begin{aligned}
\sup _{t \in[0, h]}|x(t)-\xi(t)| & \leq \varepsilon_{0}+\int_{0}^{h}\left|\bar{b}_{0,1}(x(s), s)-\bar{b}_{0,1}(\xi(s), s)\right| d s \\
& \leq \varepsilon_{0}+K \int_{0}^{h}|x(s)-\xi(s)| d s,
\end{aligned}
$$

and therefore

$$
\sup _{t \in[0, h]}|x(t)-\xi(t)| \leq \frac{\varepsilon_{0}}{1-\beta} .
$$

Similarly

$$
\sup _{t \in[h, 2 h]}|x(t)-\xi(t)| \leq \frac{\varepsilon_{0}}{1-\beta}+K \int_{h}^{2 h}|x(s)-\xi(s)| d s
$$

and

$$
\sup _{t \in[h, 2 h]}|x(t)-\xi(t)| \leq \varepsilon_{0}\left(\frac{1}{1-\beta}\right)^{2} .
$$

By repeating this procedure $n=\lceil T / h\rceil$ times (where $\lceil x\rceil$ is the smallest integer greater than $x$ ), and taking the limit as $h \downarrow 0$, one obtains (39).

Using Theorem 2, choose $I_{0}^{\prime \prime}<I_{0}^{\prime}$ such that for $I<I_{0}^{\prime \prime}$,

$$
\operatorname{Pr}\left\{\sup _{t \in[0, T]}\left|\xi_{I}(t)-\xi_{I}(0)-\int_{0}^{t} \bar{b}_{I, 1}\left(\xi_{I}(s), s\right) d s-w_{1}(t)\right|>\frac{\varepsilon_{0}}{2}\right\}<\frac{\varepsilon}{4} .
$$

By Chebyshev's inequality, we also have that

$$
\begin{aligned}
& \operatorname{Pr}\left\{\omega \in \Lambda_{I}: \sup _{t \in[0, T]}\left|\int_{0}^{t} \bar{b}_{I, 1}\left(\xi_{I}(s), s\right) d s-\int_{0}^{t} \bar{b}_{0,1}\left(\xi_{I}(s), s\right) d s\right|>\frac{\varepsilon_{0}}{2}\right\} \\
& \quad \leq\left(\frac{2}{\varepsilon_{0}}\right) \int_{D}\left|\bar{b}_{I, 1}-\bar{b}_{0,1}\right| \hat{\rho}_{I} d x d t .
\end{aligned}
$$

Choose $I_{0}^{\prime \prime \prime}<I_{0}^{\prime \prime}$ such that for $I<I_{0}^{\prime \prime \prime}$,

$$
\sup _{(x, t) \in D}\left|\bar{b}_{I, 1}-\bar{b}_{0,1}\right| \leq\left(\frac{\varepsilon_{0}}{2}\right) \frac{\varepsilon}{4 T} .
$$

Then, for $I<I_{0}^{\prime \prime \prime}$,

$$
\operatorname{Pr}\left\{\omega \in \Lambda_{I}: \sup _{t \in[0 . T]}\left|\xi_{I}(t)-\xi_{I}(0)-\int_{0}^{t} \bar{b}_{0,1}\left(\xi_{I}(s), s\right) d s-w_{1}(t)\right|>\varepsilon_{0}\right\}<\frac{\varepsilon}{2} .
$$

Since $\omega \in \Lambda_{I}$ implies that $\left(\xi_{I}(t), t\right) \in D$ for all $t$ in $[0, T]$,

$$
\operatorname{Pr}\left\{\omega \in \Lambda_{I}: \sup \left|\xi_{I}(t)-\xi(t)\right|>\varepsilon\right\}<\varepsilon / 2
$$


But $\operatorname{Pr}\left\{\Lambda_{I}\right\}>1-\varepsilon / 2$, so

$$
\operatorname{Pr}\left\{\sup _{t \in[0, T]}\left|\xi_{I}(t)-\xi(t)\right|>\varepsilon\right\}<\varepsilon .
$$

\section{THE LIMIT PROCESS}

What do we know about the limit process? It has density $\hat{\rho}_{0}(x, t)$ and forward drift $\bar{b}_{0,1}(x, t)$. By Theorem 1 , the osmotic velocity $\hat{u}_{0}$ is equal to $\bar{u}_{0,1}$. Therefore the current velocity equals $\bar{v}_{0,1}$. This, then, is just the process with averaged drifts associated with the solution to the regular Pauli equation.

How is this process related to the diffusion associated with the (spinor) Pauli equation? We will show that when the initial condition for the regular Pauli equation consists of $(2 s+1)$ parallel columns, the diffusion coincides with that associated with a Pauli spinor parallel to these columns. Otherwise, the density evolution of the limit process does not in general coincide with that of any Pauli spinor.

Assume $\psi(x, y, 0)$ is in $\mathscr{K}^{s}$. Let $\chi_{i j}(y)$ be an orthonormal basis for the spin states such that, in accordance with physics convention, the irreducible subspaces are columns in the usual matrix notation. We write the solution to the regular Pauli equation as

$$
\psi(x, y, t)=\sum_{i, j} \psi_{i j}(x, t) \chi_{i j}(y) .
$$

If we calculate $\bar{v}_{1}$, where $v$ is defined as $\operatorname{Im}(\nabla \psi / \psi)$, we find from the orthonormality of the $\chi_{i j}$ that

$$
\hat{\rho} \bar{v}_{1}=\sum_{i, j} \rho_{i j}(x) v_{i j}(x),
$$

where $v_{i j}=\operatorname{Im}\left(\nabla \psi_{i j}(x) / \psi_{i j}(x)\right)$. A similar relation holds for $\bar{u}_{1}$. These formulas are identical to those heuristically derived for the Pauli equation (9), except that now we are averaging over a matrix of drifts, rather than a vector of drifts.

If $U_{P}(t)$ and $U_{R P}(t)$ are the unitary groups for the Pauli and regular Pauli equations, and if

$$
\psi_{R P}(x, 0)_{i j}=\psi_{P}^{(j)}(x, 0)_{i},
$$

where $i$ denotes the spinor index of $\psi_{P}^{(j)}$, then

$$
\left(U_{R P}(t) \psi_{R P}\right)_{i j}=\left(U_{P}(t) \psi_{P}^{(j)}\right)_{i} .
$$

This just expresses the fact that the regular Pauli state space consists of $(2 s+1)$ orthogonal columns of Pauli spinors. Now

$$
v_{P}=\frac{\sum_{i} v_{i} \rho_{i}}{\sum \rho_{i}} \text { and } v_{R P}=\frac{\sum_{i, j} v_{i j} \rho_{i j}}{\sum_{i, j} \rho_{i j}},
$$


where we have adopted obvious notational simplifications. Clearly, if all the columns of the wavefunction for the regular Pauli equation are parallel, which is to say, if $\psi_{R P}(x, 0)_{i j}=c_{j} \psi_{P}(x, 0)_{i}$, where $\sum_{j}\left|c_{j}\right|^{2}=1$, then $v_{P}(t)=v_{R P}(t)$, $\rho_{P}(t)=\rho_{R P}(t)$, and $u_{P}(t)=u_{R P}(t)$. If, on the other hand, $\psi_{R P}(x, 0)_{i j}=$ $c_{j} \psi_{P}^{(j)}(x, 0)_{i}$ where the $\psi_{P}^{(j)}$ are not all parallel, there will not in general be any $v_{P}$ and $\rho_{P}$ corresponding to $v$ and $\rho$.

Acknowledgments. This paper is part of the author's Princeton University Ph.D. thesis. The author would like to thank his thesis advisor, Professor Edward Nelson, for his encouragement and interest in the work, and for his careful review of the manuscript.

\section{REFERENCES}

1. Fritz Bopp and Rudolf Haag, Über die Möglichkeit von Spinmodellen, Z. Naturforsch. 5a (1950), 644-653.

2. Eric A. Carlen, Conservative diffusions, Comm. Math. Phys. 94 (1984), 293-315.

3. Thaddeus George Dankel, Jr., Mechanics on manifolds and the incorporation of spin into Nelson's stochastic mechanics, Arch. Rational Mech. Anal. 37 (1970), 192-222.

4. William G. Faris, Spin correlation in stochastic mechanics, Found. Phys. 12 (1982), 1-26.

5. Imre Fényes, Eine wahrscheinlichkeitstheoretische Begründung und Interpretation der Quantenmechanik, Z. Phys. 132 (1952), 81-106.

6. H. P. McKean, Jr., Stochastic integrals, Prob. Math. Statist., vol. 5, Academic Press, 1969.

7. Edward Nelson, Quantum fluctuations, Princeton Univ. Press, Princeton, N.J., 1985.

8. Elias M. Stein, Topics in harmonic analysis, Princeton Univ. Press, Princeton, N.J., 1970.

9. Timothy C. Wallstrom, A finite energy bound on the approach of a diffusion to the zeros of its density, Proc. Amer. Math. Soc. 108 (1990), 839-843.

10. __ Ergodicity of finite-energy diffusions, Trans. Amer. Math. Soc. 318 (1990), 735-747.

11. _ On the derivation of the Schrödinger equation from stochastic mechanics, Found. Phys. Lett. 2 (1989), 113-126.

Department of Physics, Princeton University, Princeton, New Jersey 08544

Current address: 21 East Camino Real, Arcadia, California 91006 